\title{
Electrochemical sandwich immunosensor for determination of exosomes based on surface marker-mediated signal amplification.
}

\author{
Ximena Doldán ${ }^{1}$, Pablo Fagúndez ${ }^{1}$, Alfonso Cayota ${ }^{2,3}$, Justo Laíz ${ }^{1}$, Juan Pablo Tosar ${ }^{1,2 *}$ \\ ${ }^{1}$ Analytical Chemistry Unit, Nuclear Research Center, Faculty of Sciences, Universidad de la República, Uruguay. \\ ${ }^{2}$ Functional Genomics Laboratory, Institut Pasteur de Montevideo, Uruguay. ${ }^{3}$ Department of Medicine, Faculty of \\ Medicine, Universidad de la República, Uruguay.
}

\section{SUPPLEMENTARY FIGURES}
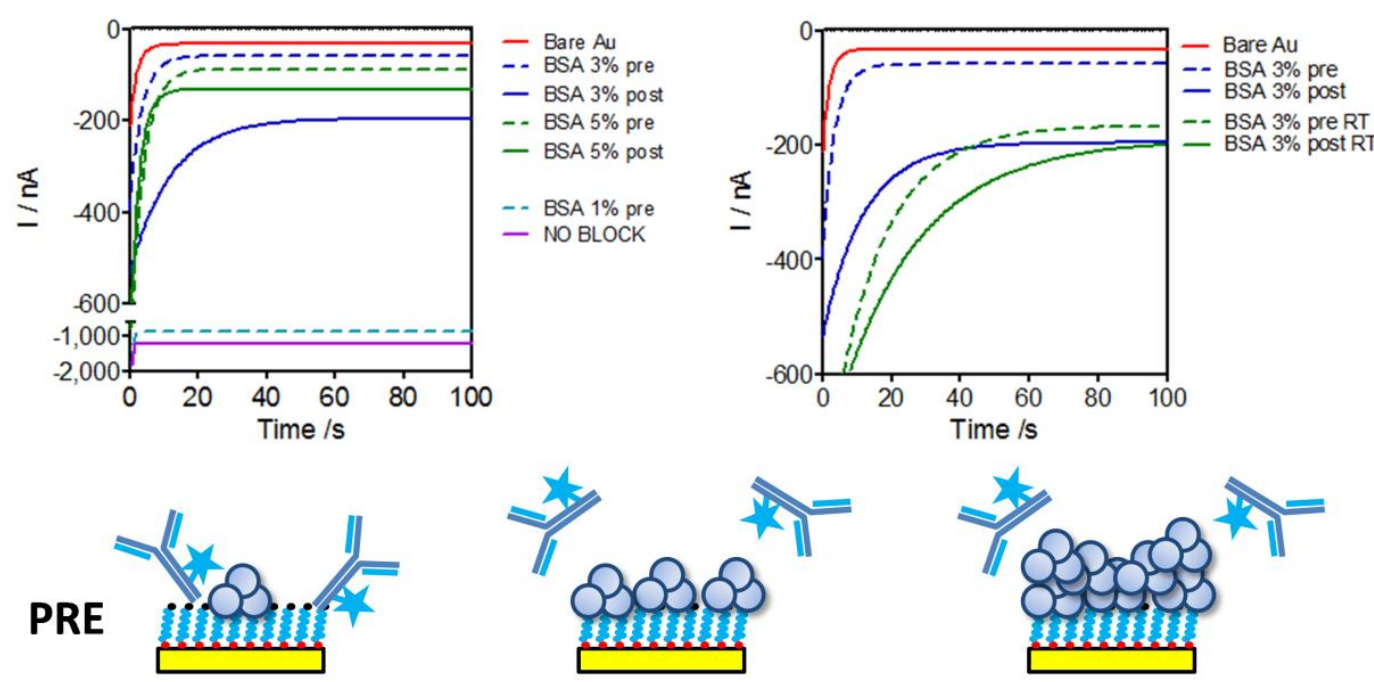

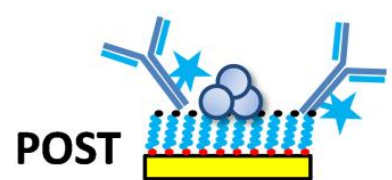

BSA 1\%

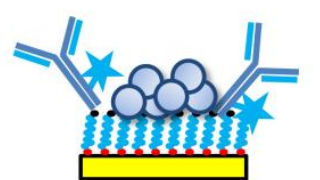

BSA 3\%

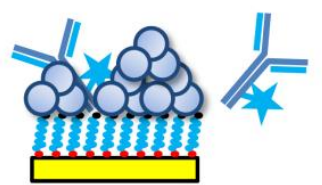

BSA 5\%

Supplementary Figure 1: Optimization of electrode blocking with BSA in Au/MUA electrodes. A) Three different BSA concentrations were tested $(1 \%, 3 \%, 5 \%$ w/v; diluted in PBS), and two configurations. "Pre" assays consisted in adding BSA before HRP-conjugated antibodies. "Post" assays were the opposite. Optimal blocking should show: i) very low current (absolute value) in the "pre" configuration, and much higher current (absolute value) in the "post" assays. This was only obtained with 3\% BSA. B) Comparison of 15 min blocking with $3 \%$ BSA at $37^{\circ} \mathrm{C}$ (blue) and room temperature (green). Dashed curves correspond to the "pre" configuration and solid curves to the "post". 


\section{$m \alpha-C D 9(h)+E X O$}

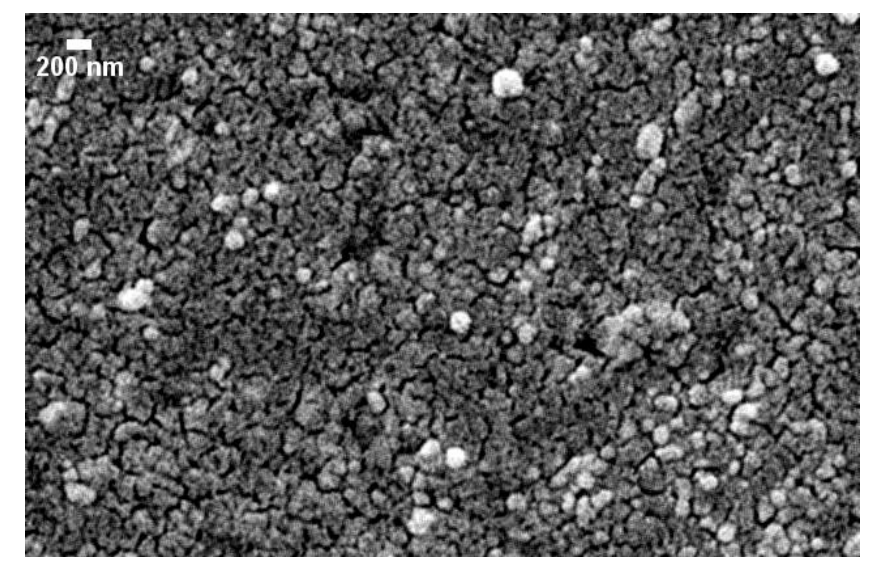

\section{$m \alpha-C D 9(h)+M V$}

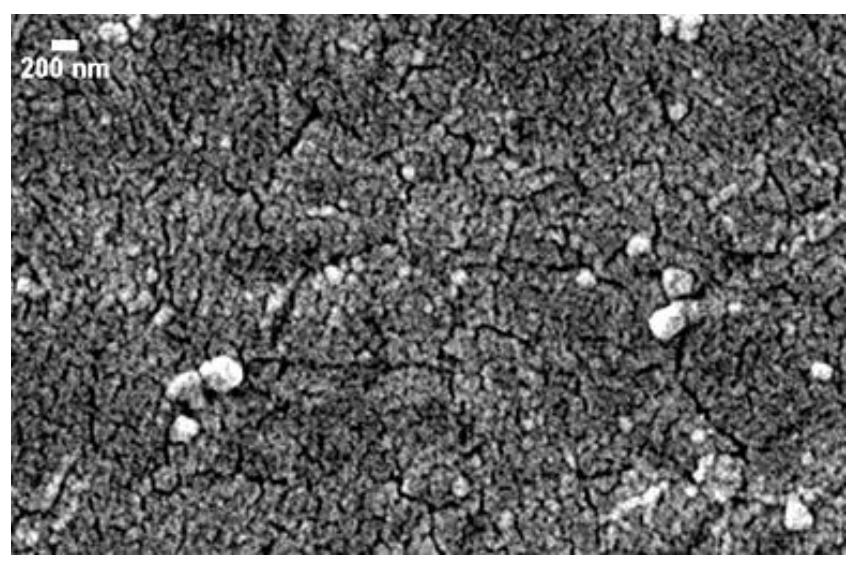

\section{$m \alpha-T S G 101(h)+E X O$}

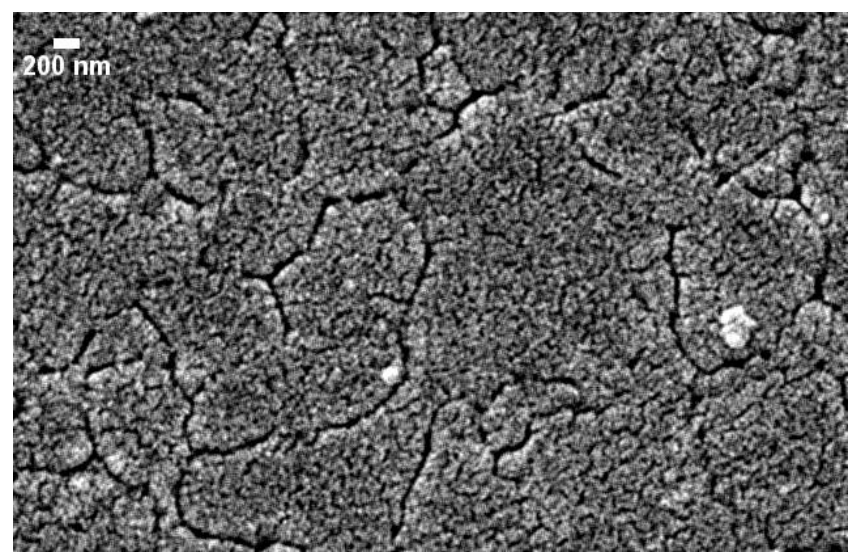

Supplementary Figure 2: Scanning electron micrograph of Au/MUA electrodes modified with mouse monoclonal $\alpha$-human CD9 antibodies (top, center) or mouse monoclonal $\alpha$-human TSG101 antibodies (bottom), after blocking with BSA and addition of exosomes (top, bottom) or microvesicles (center) at $1 \times 10^{6}$ particles / $\mu \mathrm{L}$. 


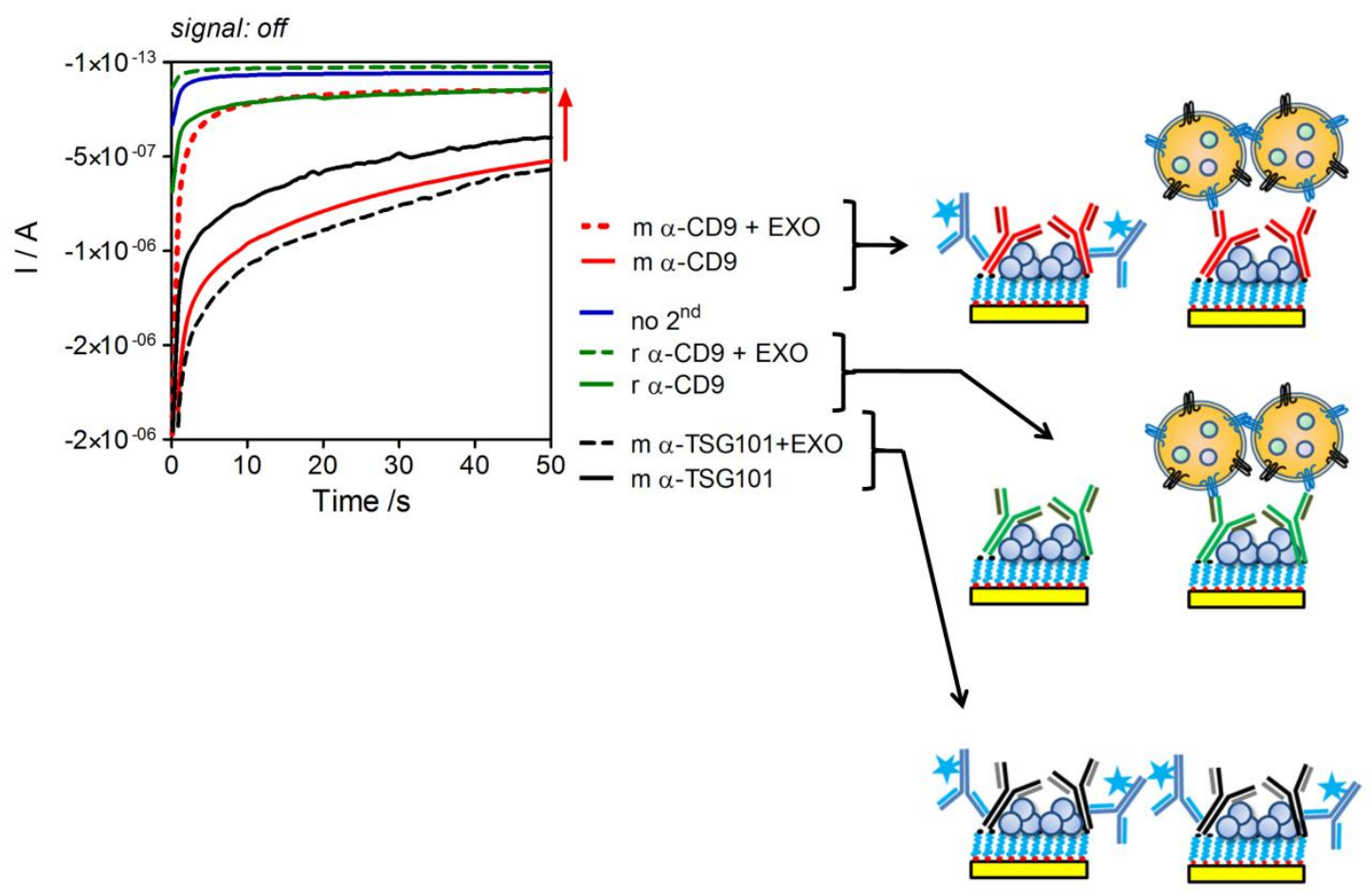

Supplementary Figure 3: evaluation of antibody specificity by the signal-off method. $\mathrm{Au} / \mathrm{MUA}$ electrodes were incubated with different capture antibodies: mouse monoclonal $\alpha$ human CD9 (red), rabbit polyclonal $\alpha$-human CD9 (green) and mouse monoclonal $\alpha$-human TSG101 (black). Then, HRP-conjugated $\alpha$-mouse IgG ABs were added and electrochemical $\mathrm{TMB}$ reduction was monitored. As a control, conjugated $\mathrm{ABs}$ were omitted (blue). 